\title{
Efeitos da Deriva de Clomazone, Aplicado isoladamente ou em Mistura com Ametryn, sobre Características Produtivas de Laranj eira 'HAMLIN"
}

\author{
Effects of Clomazone Drift, Sprayed alone or in Mixture with Ametryn, on the Productive \\ Characteristics of Hamlin Orange
}

TIMOSSI, P.C. ${ }^{2}$ e ALVES, P.L.C.A. ${ }^{3}$

\begin{abstract}
RESUMO - Este trabalho objetivou avaliar o efeito da simulação da deriva de doses crescentes do herbicida clomazone, em duas formulações, e de clomazone em mistura com ametryn, em laranjeira 'Hamlin', em dois estádios de desenvolvimento: pleno de florescimento e início da frutificação (frutos com até $2 \mathrm{~cm}$ de diâmetro). As avaliações basearam-se em possiveis alterações morfofisiológicas das plantas, com determinações do teor de clorofila total nas folhas, porcentagem de aborto de flores e frutos, além de análise tecnológica dos frutos (diâmetro transversal, teor de sólidos solúveis, acidez titulável, índice de maturação e porcentagem de suco). Observou-se que apenas a simulação de deriva de clomazone, isolado ou em mistura com ametryn, equivalente a uma aplicação direta na dose comercial (100\%), em laranjeira no estádio de desenvolvimento com frutos de até $2 \mathrm{~cm}$ de diâmetro, resultou em aborto de frutos, enquanto as derivas em menores concentrações e os demais tratamentos não resultaram em aborto de frutos em nenhum dos dois estádios da laranjeira. Os tratamentos utilizados não acarretaram qualquer alteração qualitativa no suco da laranja. Deriva de clomazone equivalente a doses acima de 50\% da dose comercial, isolado ou em mistura com ametryn, causou pontos cloróticos e necróticos na casca dos frutos.
\end{abstract}

Palavras-chave: herbicida, fitotoxicidade, citros, formulação.

\begin{abstract}
This experiment aimed to evaluate the effect of increasing clomazone (sprayed alone or in mixture with ametryn) drift simulation on quantitative and qualitative aspects of orange production when applied at two developmental plant stages: flowering and initial fruit development (fruits $2 \mathrm{~cm}$ diameter or smaller). Increasing drifts of clomazone, in two formulations, and clomazone in mixtures with ametryn were tested. Fruit morphological features, fruit color, flower and fruit abortion and leaf chlorophyll content were evaluated. Simulated drift similar to the recommended dose of clomazone and clomazone plus ametryn caused fruit abortion in citrus at the stage of fruits $2 \mathrm{~cm}$ diameter or smaller. Lower drift doses did not result in fruit abortion. Fruit diameter reduction was observed with drift off above 25\% of clomazone alone recommended rate and 50\% when in mixture with ametryn or in microcapsule formulation. Clomazone drift at $50 \%$ of the recommended rate (alone or in mixture with ametryn) caused clorotic and necrotic spots in the fruit peel. Drift did not affect juice quality in any of the treatments tested.
\end{abstract}

Key words: herbicide, phytotoxicity, citrus, formulation.

Recebido para publicação em 27/6/2000 e na forma revisada em 2/7/2001.

Eng.-Agrônomo, Mestrando em Produção Vegetal pela FCAVJ-UNESP; ${ }^{3}$ Prof. Assistente, Doutor do Dep. de Biologia Aplicada à Agropecuária da FCAVJ-UNESP. Rod. de acesso Prof. Paulo D. Castellane, s/n, 14884-900 Jaboticabal-SP. <plalves@fcav.unesp.br>

Planta Daninha, Viçosa-MG, v.19, n.2, p.295-304, 2001 


\section{INTRODUÇÃO}

Apesar das grandes produções obtidas pelo setor citrícola nos últimos anos, este tem apresentado base tecnológica baixa, uma vez que ele não tem se mostrado muito rentável, não proporcionando, conseqüentemente, o devido retorno de investimento na cultura.

Na região citrícola do Estado de São Paulo, a erradicação de pomares coincidiu principalmente com a expansão das áreas de plantio de cana-de-açúcar, fazendo com que uma cultura fosse trocada por outra ou então que elas se tornassem freqüentemente vizinhas.

O setor sucroalcooleiro vem procurando otimizar suas aplicações de defensivos como uma forma de reduzir despesas. Uma das formas de otimização é por meio das aplicações aéreas, que vêm sendo muito utilizadas para herbicidas e maturadores, mas que aumenta o risco de deriva desses produtos em culturas que não eram alvo (Gelmini, 1988). Ao se fazer a escolha de um determinado herbicida, devemse avaliar os riscos e benefícios do uso do produto, considerando a forma de uso, a importância econômica e a presença de culturas vizinhas suscetíveis (Auch \& Arnold, 1978). O objetivo da aplicação de um herbicida é colocar a quantidade certa de ingrediente ativo no alvo desejado, com a máxima eficiência e da maneira mais econômica possivel, sem afetar o ambiente (Durigan, 1989). A ocorrência de riscos de deriva de produtos seletivos à cultura de cana-de-açúcar em culturas suscetíveis como a de citros vem causando sérios problemas, devido a pressupostos erros na tecnologia de aplicação.

Segundo Matuo (1990), a aplicação de defensivos, principalmente a aérea, quando feita na presença de ventos com velocidade acima de $5 \mathrm{~km} \mathrm{~h}^{-1}$, pode provocar o carregamento de partículas menores do produto por longas distâncias (deriva), provocando danos, em alguns casos, às culturas ou plantas que não eram seu alvo. Depreende-se, assim, que o herbicida deve atingir de modo eficaz a planta daninha a ser controlada, uma vez que a ocorrência de deriva, além de reduzir a eficiência da aplicação (Gelmini, 1988), coloca em risco culturas vizinhas suscetiveis. Gotas pequenas podem ser transportadas em condições variáveis de vento e luminosidade, particularmente em condições de inversão térmica, atingindo assim áreas agrícolas adjacentes, sendo possível verificar sintomas de injúrias causados por deriva de herbicidas a quilômetros de distância do local de aplicação (Yates et al., 1978).

Para Bayley \& Kapusta (1993), a iminente possibilidade de ocorrência de injúrias em culturas, devido à deposição de herbicidas em áreas "não alvo", tem levado à condução de várias pesquisas envolvendo o conceito de "deriva simulada”, principalmente em culturas nas quais as aplicações são realizadas com equipamento aéreo.

Tendo em vista o que foi exposto, este trabalho objetivou avaliar o efeito da deriva simulada de clomazone, em duas formulações, e de clomazone em mistura com ametryn, herbicidas estes normalmente utilizados no controle de plantas daninhas em pré-emergência e pós-emergência na cultura da canade-açúcar, sobre características quantitativas e qualitativas de frutos de laranjeira (Citrus sinensis).

\section{MATERIAL E MÉTODOS}

O estudo constou de dois ensaios instalados em área de produção de laranja (Citrus sinensis), no município de Taiúva, SP.

Os tratamentos experimentais constaram da aplicação de doses crescentes de clomazone, isolado ou em mistura com ametryn (até que essas atingissem a dose recomendada comercialmente para o controle de plantas daninhas), em dois estádios de desenvolvimento das laranjeiras, a saber: pleno de florescimento e início da frutificação (frutos com até $2 \mathrm{~cm}$ de diâmetro). Estes estádios de aplicação corresponderam aos dois ensaios, instalados em setembro de 1998 e conduzidos simultaneamente até abril de 1999. A descrição dos tratamentos experimentais utilizados nos dois ensaios encontra-se na Tabela 1.

A aplicação dos herbicidas foi realizada com pulverizador costal à pressão constante (ar comprimido), munido de um bico XR1 1002 e regulado para um gasto de volume de calda de $200 \mathrm{~L} \mathrm{ha}^{-1}$. Essa aplicação foi realizada a um metro de distância das árvores, direcionada 
para a porção mediana destas, resultando em uma faixa tratada de $0,45 \mathrm{~m}^{2}$ por aplicação. Como os ensaios compreenderam diferentes estádios de desenvolvimento das laranjeiras, no experimento foram feitas duas aplicações, cujas datas e condições climáticas encontramse descritas na Tabela 2 .

As parcelas experimentais constaram das faixas de aplicação, sendo aplicadas três faixas em faces opostas de uma mesma planta, correspondendo, cada qual, a uma repetição do tratamento. Como testemunha, foram consideradas três faixas por planta não-tratada.

O experimento foi instalado no campo, segundo o delineamento experimental de blocos ao acaso, com os tratamentos em três repetições, as quais foram obtidas pelas médias de observações efetuadas nas faixas tratadas, de acordo com o ensaio.

No primeiro ensaio, que correspondeu ao pleno florescimento das plantas, foram utilizados dois ramos locados na faixa tratada, sendo estes demarcados com fitilho, nos quais foram avaliados os efeitos dos tratamentos. No segundo ensaio, na frutificação das plantas, foram marcados com tinta branca 10 frutos por faixa tratada, nos quais foram avaliados os efeitos dos tratamentos.
Em cada ensaio, os efeitos dos tratamentos foram avaliados aos 15, 30, 60, 90 e 120 dias após aplicação (DAA), quando foram efetuadas contagens de flores e/ou frutos na faixa tratada. No primeiro ensaio foi feita uma contagem prévia das flores existentes nos ramos, antes de estes serem expostos aos tratamentos, a fim de se constatar a variabilidade inicial e isolar os efeitos desta sobre a porcentagem final de aborto. Com os resultados dessas contagens, foi calculada a taxa porcentual de aborto de flores e de frutos acumulada aos 120 DAA em resposta aos tratamentos. Nestas mesmas ocasiões, foram feitas determinações dos teores relativos de clorofila total em folhas tomadas ao acaso no terço final dos ramos e no terço médio das folhas nas faixas tratadas, utilizando um clorofilômetro manual (Minolta, mod. SPAD 502). Nessas ocasiões, foram acompanhadas quaisquer alterações morfofisiológicas nas folhas e nos frutos resultantes dos tratamentos experimentais.

Aos 150 DAA, para o ensaio em pleno florescimento, foram efetuadas medições do diâmetro transversal de três frutos remanescentes na faixa tratada, utilizando-se, para isso, um paquímetro de precisão. O mesmo foi feito aos 120 DAA para o ensaio com frutos de até $2 \mathrm{~cm}$ de diâmetro.

Tabela 1 - Descrição dos tratamentos experimentais, com as respectivas doses dos ingredientes ativos (i.a.) e dos produtos comerciais, e suas correspondências com a concentração porcentual, aplicados em laranjeira Hamlin (\% v/v). Taiúva, 1998

\begin{tabular}{|c|c|c|c|c|}
\hline \multirow{2}{*}{$\mathrm{N}^{\mathrm{o}}$} & \multirow{2}{*}{ Tratamentos } & \multicolumn{2}{|c|}{ Dose } & \multirow{2}{*}{$\begin{array}{c}\text { Concentração } \\
(\% \mathrm{v} / \mathrm{v})\end{array}$} \\
\hline & & i.a. $\left(\mathrm{kg} \mathrm{ha}^{-1}\right)$ & p.c. $\left(\mathrm{kg}\right.$ ou L ha $\left.{ }^{-1}\right)$ & \\
\hline 1 & Clomazone $* \mathrm{C}-3,12$ & 0,031 & 0,062 & 3,12 \\
\hline 2 & Clomazone - C-6,25 & 0,062 & 0,125 & 6,25 \\
\hline 3 & Clomazone - C-12,5 & 0,125 & 0,250 & 12,50 \\
\hline 4 & Clomazone - C-25 & 0,250 & 0,500 & 25,00 \\
\hline 5 & Clomazone - C -50 & 0,500 & 1,000 & 50,00 \\
\hline 6 & Clomazone - C-100 & 1,000 & 2,000 & 100,00 \\
\hline 7 & Clomazone+ametryn $* *$ CA-1,06 & $0,015+0,023$ & 0,078 & 1,06 \\
\hline 8 & Clomazone + ametryn - CA-3,12 & $0,031+0,047$ & 0,156 & 3,12 \\
\hline 9 & Clomazone + ametryn - CA- 6,25 & $0,062+0,094$ & 0,312 & 6,25 \\
\hline 10 & Clomazone + ametryn - CA- 12,5 & $0,125+0,187$ & 0,625 & 12,50 \\
\hline 11 & Clomazone + ametryn - CA-25 & $0,250+0,375$ & 1,250 & 25,00 \\
\hline 12 & Clomazone + ametryn - CA-50 & $0,500+0,750$ & 2,500 & 50,00 \\
\hline 13 & Clomazone + ametryn - CA-100 & $1000+1500$ & 5,000 & 100,00 \\
\hline 14 & Clomazone $* * *$ - CM & 1000 & 2,770 & 100,00 \\
\hline 15 & Testemunha & ------- & ------- & ------- \\
\hline
\end{tabular}

* Gamit ** Sinerge *** Gamit microencapsulado. 
Tabela 2 - Datas e condições climáticas por ocasião das aplicações dos tratamentos experimentais. Taiúva, 1998

\begin{tabular}{|c|c|c|c|c|c|c|c|}
\hline \multirow{2}{*}{ Data } & \multicolumn{2}{|c|}{ Horário } & \multirow{2}{*}{$\begin{array}{c}\text { Temp. ar } \\
\left({ }^{\circ} \mathrm{C}\right)\end{array}$} & \multirow{2}{*}{$\begin{array}{l}\text { Umidade } \\
\text { do solo }\end{array}$} & \multirow{2}{*}{$\begin{array}{l}\text { U.R. } \\
(\%)\end{array}$} & \multirow{2}{*}{$\begin{array}{l}\text { Cobertura do } \\
\text { céu }(\%)\end{array}$} & \multirow{2}{*}{ vento } \\
\hline & início & fim & & & & & \\
\hline $02 / 09$ & $10 \mathrm{~h} 40 \mathrm{~min}$ & $11 \mathrm{~h} 05 \mathrm{~min}$ & 27,5 & úmido & 65 & aberto & forte* \\
\hline $30 / 09$ & $15 \mathrm{~h} 55 \mathrm{~min}$ & $16 \mathrm{~h} 15 \mathrm{~min}$ & 29,0 & úmido & 81 & 40 & ausente \\
\hline
\end{tabular}

* Rajadas.

Ao término do período experimental, por ocasião da colheita dos frutos, estes foram submetidos a análises tecnológicas, seguindo padrões industriais, que foram: diâmetro transversal dos frutos, teor de sólidos solúveis, acidez titulável e índice de maturação ("ratio"), segundo Tressler \& Joslyn (1961), e porcentagem de suco.

Os resultados obtidos foram submetidos à análise de variância pelo teste $\mathrm{F}$ e as médias foram comparadas pelo teste de Tukey a 5\% de probabilidade. Para fins de normalização, os resultados expressos em porcentagem foram transformados para arc sen $\sqrt{\mathrm{x}}$ antes de serem submetidos à análise de variância.

\section{RESULTADOS E DISCUSSÃO}

\section{Ensaio com botão floral}

Em avaliação prévia do número de flores por ramo (Tabela 3), constatou-se que aqueles que receberiam tratamento com a metade da dose comercial de clomazone (C-50) naturalmente já apresentavam menor número de flores do que aqueles que receberiam $25 \%$ da dose do mesmo produto (C-25). Contudo, aos 15 dias após aplicação (DAA) observou-se que não houve diferença na porcentagem de aborto de flores em decorrência da aplicação dos tratamentos.

Aos 30 DAA, verificou-se aumento acentuado na taxa de flores abortadas em todos os tratamentos, aumento este de ocorrência natural, uma vez que na testemunha a taxa também foi alta. Mas aqueles ramos tratados com a maior dose de clomazone (C-100) apresentaram taxa de aborto superior à dos ramos tratados com maior dose da mistura de clomazone com ametryn (CA-100), sem que ambos diferissem da testemunha (sem aplicação). Nessa ocasião, foram observados os primeiros sintomas de fitotoxicidade nos frutos ou nas folhas.

Aos 45 DAA, quando já havia frutos formados nos ramos, verificou-se que a taxa de aborto de frutos nos tratamentos que empregaram 12,5 e $25 \%$ de clomazone $(\mathrm{C}-12,5)$ e clomazone + ametryn (CA-50), respectivamente, foi maior do que a dos ramos tratados com $3,12 \%$ de clomazone $(\mathrm{C}-3,12)$, sem que nenhum dos tratamentos tenha diferenciado da testemunha.

Aos 60 DAA, quando os frutos se encontravam com $1 \mathrm{~cm}$ de diâmetro, não mais se constatou diferença na taxa de aborto em decorrência dos tratamentos. O mesmo comportamento foi observado aos 90 DAA, à exceção do tratamento empregando $6,25 \%$ de clomazone $(\mathrm{C}-6,25)$, que resultou em maior taxa de aborto do que a de todos os tratamentos, incluindo a testemunha.

Aos 120 DAA, quando se calculou a taxa acumulada de aborto, verificou-se que a maior dose de clomazone resultou em maior taxa de aborto de flores/frutos do que as duas menores doses do mesmo produto e a dose de $25 \%$ da mistura de clomazone + ametryn; isso porque nesse tratamento não se constatou nenhum fruto nos ramos. Nessa ocasião pôde-se observar que a taxa de aborto natural em laranjeira Hamlin foi muito elevada (acima de 90\%), ou seja, um ramo contendo em média 30 flores produziu, após quatro meses, dois frutos.

Com relação à análise do teor total de clorofila (Tabela 4), observou-se aos 15 e 30 DAA que os tratamentos empregando doses superiores a $12,5 \%$ da mistura de clomazone com ametryn resultaram em menor teor de clorofila do que as duas menores doses desse produto e da testemunha, enquanto a aplicação de clomazone isolado, independentemente da concentração, incluindo formulação microencapsulada (CM), não diferiu da testemunha. 
Efeitos da deriva de clomazone, aplicado isoladamente ou em mistura ...

Tabela 3 - Número de flores por ramo, anterior à aplicação dos tratamentos (prévia), e porcentagens de flores/frutos abortados no decorrer do período experimental e em função dos tratamentos experimentais em laranjeira 'Hamlin'. Taiúva, 1998

\begin{tabular}{|c|c|c|c|c|c|c|c|}
\hline \multirow{2}{*}{ Tratamentos } & \multicolumn{7}{|c|}{ Porcentagem de frutos abortados } \\
\hline & Prévia & $15 \mathrm{DAA}$ & $30 \mathrm{DAA}$ & 45 DAA & $60 \mathrm{DAA}$ & 90 DAA & $120 \mathrm{DAA}$ \\
\hline $\mathrm{C}-3,12$ & $29,9 \mathrm{AB}$ & $39,2 \mathrm{~A}$ & $57,2 \mathrm{AB}$ & $16,2 \mathrm{~B}$ & $0,0 \mathrm{~A}$ & $0,0 \mathrm{~B}$ & $60,1 \mathrm{C}$ \\
\hline$C-6,25$ & $26,8 \mathrm{AB}$ & $26,6 \mathrm{~A}$ & $52,8 \mathrm{AB}$ & $52,5 \mathrm{AB}$ & $15,0 \mathrm{~A}$ & $15,0 \mathrm{~A}$ & $69,9 \mathrm{BC}$ \\
\hline $\mathrm{C}-12,5$ & $28,9 \mathrm{AB}$ & $26,9 \mathrm{~A}$ & $53,9 \mathrm{AB}$ & $75,0 \mathrm{~A}$ & $7,5 \mathrm{~A}$ & $0,0 \mathrm{~B}$ & $82,6 \mathrm{AB}$ \\
\hline$C-25$ & $44,1 \mathrm{~A}$ & $26,4 \mathrm{~A}$ & $51,3 \mathrm{AB}$ & $71,2 \mathrm{AB}$ & $0,0 \mathrm{~A}$ & $0,0 \mathrm{~B}$ & $79,6 \mathrm{ABC}$ \\
\hline$C-50$ & $14,2 \mathrm{~B}$ & $34,5 \mathrm{~A}$ & $60,9 \mathrm{AB}$ & $49,8 \mathrm{AB}$ & $20,9 \mathrm{~A}$ & $0,0 \mathrm{~B}$ & $82,3 \mathrm{AB}$ \\
\hline $\mathrm{C}-100$ & $22,2 \mathrm{AB}$ & $38,1 \mathrm{~A}$ & $77,1 \mathrm{~A}$ & $45,0 \mathrm{AB}$ & $0,0 \mathrm{~A}$ & $0,0 \mathrm{~B}$ & $90,0 \mathrm{~A}$ \\
\hline $\mathrm{CA}-1,06$ & $34,4 \mathrm{AB}$ & $37,8 \mathrm{~A}$ & $57,5 \mathrm{AB}$ & $46,6 \mathrm{AB}$ & $0,0 \mathrm{~A}$ & $0,0 \mathrm{~B}$ & $74,9 \mathrm{ABC}$ \\
\hline $\mathrm{CA}-3,12$ & $37,8 \mathrm{AB}$ & $34,0 \mathrm{~A}$ & $54,0 \mathrm{AB}$ & $62,7 \mathrm{AB}$ & $0,0 \mathrm{~A}$ & $0,0 \mathrm{~B}$ & $73,1 \mathrm{ABC}$ \\
\hline $\mathrm{CA}-6,25$ & $30,6 \mathrm{AB}$ & $35,2 \mathrm{~A}$ & $56,3 \mathrm{AB}$ & $55,6 \mathrm{AB}$ & $0,0 \mathrm{~A}$ & $0,0 \mathrm{~B}$ & $73,5 \mathrm{ABC}$ \\
\hline $\mathrm{CA}-12,5$ & $33,3 \mathrm{AB}$ & $28,9 \mathrm{~A}$ & $68,5 \mathrm{AB}$ & $20,0 \mathrm{AB}$ & $0,0 \mathrm{~A}$ & $0,0 \mathrm{~B}$ & $73,5 \mathrm{ABC}$ \\
\hline $\mathrm{CA}-25$ & $27,4 \mathrm{AB}$ & $20,1 \mathrm{~A}$ & $55,4 \mathrm{AB}$ & $49,3 \mathrm{AB}$ & $7,5 \mathrm{~A}$ & $0,0 \mathrm{~B}$ & $70,0 \mathrm{BC}$ \\
\hline $\mathrm{CA}-50$ & $30,4 \mathrm{AB}$ & $27,2 \mathrm{~A}$ & $49,0 \mathrm{AB}$ & $76,6 \mathrm{~A}$ & $0,0 \mathrm{~A}$ & $0,0 \mathrm{~B}$ & $82,7 \mathrm{AB}$ \\
\hline $\mathrm{CA}-100$ & $40,4 \mathrm{AB}$ & $12,1 \mathrm{~A}$ & $45,1 \mathrm{~B}$ & $62,2 \mathrm{AB}$ & $5,9 \mathrm{~A}$ & $0,0 \mathrm{~B}$ & $75,9 \mathrm{ABC}$ \\
\hline $\mathrm{CM}$ & $28,3 \mathrm{AB}$ & $15,0 \mathrm{~A}$ & $48,5 \mathrm{AB}$ & $65,0 \mathrm{AB}$ & $0,0 \mathrm{~A}$ & $0,0 \mathrm{~B}$ & $74,8 \mathrm{ABC}$ \\
\hline Testemunha & $22,1 \mathrm{AB}$ & $24,6 \mathrm{~A}$ & $64,4 \mathrm{AB}$ & $33,1 \mathrm{AB}$ & $0,0 \mathrm{~A}$ & $0,0 \mathrm{~B}$ & $71,8 \mathrm{ABC}$ \\
\hline F blocos & $2,65^{\mathrm{NS}}$ & $0,76^{\mathrm{NS}}$ & $0,77^{\mathrm{NS}}$ & $0,57^{\mathrm{NS}}$ & $0,63^{\mathrm{NS}}$ & $1,00^{\mathrm{NS}}$ & $0,17^{\mathrm{NS}}$ \\
\hline F trat. & $1,99 *$ & $2,18 *$ & $2,05 *$ & $2,52 * *$ & $1,34^{\mathrm{NS}}$ & $2,50 * *$ & $3,27 * *$ \\
\hline $\mathrm{CV}(\%)$ & 43,57 & 48,26 & 24,89 & 54,08 & 363,61 & 600,00 & 12,68 \\
\hline DMS & 26,56 & 27,84 & 28,68 & 57,1 & 27,92 & 12,18 & 19,47 \\
\hline
\end{tabular}

${ }^{1}$ Dados transformados para arc sen $\sqrt{ } \mathrm{x}$; DAA $=$ dias após a aplicação

NS não-significativo pelo teste $\mathrm{F}$; * significativo a $5 \%$ de probabilidade; ** significativo a $1 \%$ de probabilidade.

Tabela 4 - Teor relativo de clorofila total em folhas de laranjeira 'Hamlin' em resposta aos tratamentos aplicados no estádio de botão floral. Taiúva, 1998

\begin{tabular}{|l|c|c|c|c|c|c|}
\hline \multirow{2}{*}{ Tratamentos } & \multicolumn{7}{|c|}{ Teor relativo de clorofila total nas folhas } \\
\cline { 2 - 7 } & $15 \mathrm{DAA}$ & $30 \mathrm{DAA}$ & $45 \mathrm{DAA}$ & $60 \mathrm{DAA}$ & $90 \mathrm{DAA}$ & $120 \mathrm{DAA}$ \\
\hline $\mathrm{C}-3,12$ & $40,3 \mathrm{AB}$ & $41,0 \mathrm{~A}$ & $49,7 \mathrm{~A}$ & $55,4 \mathrm{AB}$ & $61,6 \mathrm{~A}$ & $62,3 \mathrm{~A}$ \\
$\mathrm{C}-6,25$ & $42,3 \mathrm{~A}$ & $48,8 \mathrm{~A}$ & $44,7 \mathrm{AB}$ & $59,1 \mathrm{~A}$ & $56,6 \mathrm{ABC}$ & $63,5 \mathrm{~A}$ \\
$\mathrm{C}-12,5$ & $38,8 \mathrm{ABC}$ & $34,2 \mathrm{AB}$ & $41,1 \mathrm{AB}$ & $42,6 \mathrm{ABC}$ & $57,4 \mathrm{ABC}$ & $63,5 \mathrm{~A}$ \\
$\mathrm{C}-25$ & $38,7 \mathrm{ABC}$ & $47,2 \mathrm{~A}$ & $45,1 \mathrm{AB}$ & $50,9 \mathrm{AB}$ & $59,0 \mathrm{AB}$ & $62,8 \mathrm{~A}$ \\
$\mathrm{C}-50$ & $41,0 \mathrm{AB}$ & $33,2 \mathrm{AB}$ & $37,6 \mathrm{ABC}$ & $52,2 \mathrm{AB}$ & $55,6 \mathrm{ABC}$ & $64,3 \mathrm{~A}$ \\
$\mathrm{C}-100$ & $41,0 \mathrm{AB}$ & $36,4 \mathrm{AB}$ & $31,9 \mathrm{ABCD}$ & $31,0 \mathrm{BCD}$ & $48,9 \mathrm{ABC}$ & $58,5 \mathrm{AB}$ \\
\hline $\mathrm{CA}-1,06$ & $42,5 \mathrm{~A}$ & $47,4 \mathrm{~A}$ & $49,1 \mathrm{~A}$ & $56,7 \mathrm{~A}$ & $58,9 \mathrm{AB}$ & $61,4 \mathrm{AB}$ \\
$\mathrm{CA}-3,12$ & $41,0 \mathrm{AB}$ & $43,0 \mathrm{~A}$ & $49,2 \mathrm{~A}$ & $54,2 \mathrm{AB}$ & $59,6 \mathrm{AB}$ & $61,9 \mathrm{AB}$ \\
$\mathrm{CA}-6,25$ & $38,3 \mathrm{ABC}$ & $33,0 \mathrm{AB}$ & $37,6 \mathrm{ABC}$ & $46,4 \mathrm{AB}$ & $50,1 \mathrm{ABC}$ & $63,5 \mathrm{~A}$ \\
$\mathrm{CA}-12,5$ & $35,0 \mathrm{BCD}$ & $31,2 \mathrm{ABC}$ & $31,0 \mathrm{ABCD}$ & $30,8 \mathrm{BCD}$ & $57,4 \mathrm{ABC}$ & $53,5 \mathrm{~A}$ \\
$\mathrm{CA}-25$ & $30,4 \mathrm{D}$ & $11,6 \mathrm{C}$ & $22,6 \mathrm{BCD}$ & $39,3 \mathrm{ABCD}$ & $39,5 \mathrm{BC}$ & $46,6 \mathrm{AB}$ \\
$\mathrm{CA}-50$ & $31,5 \mathrm{CD}$ & $16,8 \mathrm{AC}$ & $16,5 \mathrm{CD}$ & $20,7 \mathrm{CD}$ & $39,6 \mathrm{BC}$ & $40,9 \mathrm{~B}$ \\
$\mathrm{CA}-100$ & $29,9 \mathrm{D}$ & $12,5 \mathrm{C}$ & $9,0 \mathrm{D}$ & $16,7 \mathrm{D}$ & $37,3 \mathrm{C}$ & $46,8 \mathrm{AB}$ \\
\hline CM & $38,5 \mathrm{ABC}$ & $33,0 \mathrm{AB}$ & $35,0 \mathrm{ABC}$ & $52,2 \mathrm{AB}$ & $53,6 \mathrm{ABC}$ & $56,0 \mathrm{AB}$ \\
\hline Testemunha & $39,0 \mathrm{AB}$ & $41,8 \mathrm{~A}$ & $49,1 \mathrm{~A}$ & $55,5 \mathrm{AB}$ & $50,2 \mathrm{ABC}$ & $61,9 \mathrm{AB}$ \\
\hline F. blocos & $1,42^{\mathrm{NS}}$ & $0,47^{\mathrm{NS}}$ & $1,19 \mathrm{NS}$ & $1,09 \mathrm{NS}$ & $2,25 *$ & $0,55 \mathrm{NS}$ \\
F trat. & $7,69 * *$ & $8,49 * *$ & $6,33 * *$ & $6,99 * *$ & $3,59 * *$ & $3,01 * *$ \\
CV(\%) & 12,66 & 38,44 & 43,26 & 36,8 & 25,31 & 23,68 \\
DMS & 7,42 & 20,26 & 24,52 & 25,2 & 20,51 & 21,19 \\
\hline
\end{tabular}

${ }^{1}$ Dados transformados para arc sen $\sqrt{\mathrm{x}}$; DAA $=$ dias após a aplicação.

NS não-significativo pelo teste $\mathrm{F}$; * significativo a $5 \%$ de probabilidade; ** significativo a $1 \%$ de probabilidade. 
Já aos 45 DAA, observou-se a recuperação das folhas tratadas com $25 \%$ da mistura clomazone + ametryn; seu teor de clorofila não mais diferiu dos tratamentos empregando clomazone isolado, mas ainda se mostrou inferior ao das duas menores doses da mistura e ao da testemunha. Aos 60 DAA essa dose da mistura resultou em teor de clorofila semelhante ao da testemunha, ao das menores doses da mistura e ao do clomazone isolado.

Aos 90 DAA, observou-se plena recuperação das folhas atingidas com até $50 \%$ da mistura, enquanto as atingidas com 100\% apresentavam-se ainda cloróticas. Esse comportamento de recuperação no teor de clorofila nas folhas deve-se, provavelmente, à ressintese nas folhas, não mais se observando diferenças entre os teores de clorofila nos tratamentos com a testemunha.

A análise tecnológica dos frutos (Tabela 5) revelou que aqueles atingidos com clomazone a 25\% apresentavam maior diâmetro do que os atingidos com $50 \%$ do mesmo produto isolado e dele a 50 e $100 \%$ em mistura com ametryn, sem contudo diferirem da testemunha. Aos 150 DAA os frutos tratados com clomazone a $50 \%$ apresentavam menor diâmetro do que os frutos das plantas testemunhas. Por ocasião da colheita, verificou-se que esses frutos, além de apresentarem menor diâmetro que os da testemunha, mostraram-se menores que os tratados com clomazone em mistura com ametryn até $12,5 \%$. Entretanto, não se observou qualquer efeito dos tratamentos sobre a porcentagem de suco contida nestes frutos, e frutos atingidos com clomazone isoladamente tenderam a apresentar maior índice de acidez titulável que os demais tratamentos, sem que isso se refletisse em alterações significativas no teor de sólidos solúveis ('brix) e, conseqüentemente, no "ratio".

Segundo Figueiredo (1991), citado por Rodriguez et al. (1991), a produtividade da laranjeira cv. Hamlin é excelente, podendo alcançar mais de $300 \mathrm{~kg}$ de frutos por planta. Ainda, considera-se como o cultivar mais produtivo, possuindo frutos de forma ligeiramente ovalada, quase esférica, com casca de cor laranja-clara, de espessura fina. Em média, tem $41 \%$ do peso do fruto em suco, com teores de ${ }^{\circ}$ Brix de $12 \%$, acidez titulável de $0,96 \%$ e "ratio" de 12,5 , características essas que fazem com que este cultivar assuma grande importância para a indústria de suco concentrado.

\section{Ensaio com frutos de até $2 \mathrm{~cm}$ de diâmetro}

Quando os produtos foram aplicados em ramos contendo 10 frutos recém-formados com até $2 \mathrm{~cm}$ de diâmetro (Tabela 6), não se observou qualquer alteração na taxa de aborto em decorrência dos tratamentos até os 60 DAA, embora as maiores doses de clomazone isolado ou em mistura com ametryn tendessem a proporcionar taxas de aborto mais elevadas. Essa tendência só se mostrou significativa aos 90 DAA, quando a maior dose de clomazone isolado resultou em taxa de aborto maior do que as dos demais tratamentos.

Com relação ao teor relativo de clorofila total nas folhas, não se observou qualquer alteração no seu teor em decorrência dos seus tratamentos, uma vez que esses não diferiram da testemunha até os 120 DAA. Esses resultados se devem provavelmente ao fato de os tratamentos terem sido aplicados em folhas já totalmente expandidas, e os ramos não estariam emitindo mais novas folhas.

A análise tecnológica dos frutos (Tabela 7) demonstrou que aos 120 DAA a simulação da deriva do clomazone isolado em doses acima de $25 \%$ e acima de $50 \%$ de clomazone em mistura com ametryn, bem como na formulação microencapsulada, resultou em frutos com menor diâmetro do que os das testemunhas, e esse comportamento se manteve até a ocasião da colheita dos frutos. Contudo, a porcentagem de suco contido nesses frutos só se mostrou menor naqueles tratados com clomazone a $100 \%$, sem que qualquer tratamento resultasse em alterações qualitativas nos sucos, ou seja, •brix, acidez titulável e "ratio".

\section{Observações visuais}

Observou-se visualmente que, quando o clomazone foi aplicado isoladamente a partir de $25 \%$ (C-25) nos ramos florescidos, os frutos formados apresentaram-se cloróticos, com algumas folhas na porção apical dos ramos também cloróticas; o grau dessa clorose aumentou com a dose utilizada, chegando os frutos a ficarem totalmente cloróticos quando se empregou $100 \%$ da dose comercial. 
Efeitos da deriva de clomazone, aplicado isoladamente ou em mistura ...

Tabela 5 - Resultados da análise tecnológica de frutos de laranjeira 'Hamlin' em resposta aos tratamentos, quando aplicados no estádio de botão floral. Taiúva, 1998

\begin{tabular}{|c|c|c|c|c|c|c|}
\hline \multirow{2}{*}{ Tratamentos } & \multicolumn{2}{|c|}{ Diâmetro $(\mathrm{cm})$} & \multirow{2}{*}{$\% \mathrm{Suco}^{1}$} & \multirow{2}{*}{${ }^{\circ}$ Brix } & \multirow{2}{*}{ Acidez titulável } & \multirow{2}{*}{ Ratio } \\
\hline & $150 \mathrm{DAA}$ & $240 \mathrm{DAA}$ & & & & \\
\hline$C-3,12$ & $4,5 \mathrm{AB}$ & $5,4 \mathrm{AB}$ & $45,2 \mathrm{~A}$ & $9,9 \mathrm{~A}$ & $1,32 \mathrm{~A}$ & $7,5 \mathrm{~A}$ \\
\hline$C-6,25$ & $4,3 \mathrm{BC}$ & $5,4 \mathrm{AB}$ & $44,6 \mathrm{~A}$ & $8,6 \mathrm{~A}$ & $1,02 \mathrm{AB}$ & $8,4 \mathrm{~A}$ \\
\hline$C-12,5$ & $4,3 \mathrm{BC}$ & ---- & ---- & ---- & ---- & ---- \\
\hline$C-25$ & $5,0 \mathrm{~A}$ & $5,6 \mathrm{~A}$ & $46,3 \mathrm{~A}$ & $9,2 \mathrm{~A}$ & $0,91 \mathrm{~B}$ & $10,1 \mathrm{~A}$ \\
\hline$C-50$ & $3,7 \mathrm{C}$ & $4,8 \mathrm{~B}$ & $46,8 \mathrm{~A}$ & $9,4 \mathrm{~A}$ & $1,16 \mathrm{AB}$ & $8,1 \mathrm{~A}$ \\
\hline $\mathrm{C}-100$ & ---- & ---- & ---- & --- & ---- & ---- \\
\hline $\mathrm{CA}-1,06$ & $4,5 \mathrm{AB}$ & $5,5 \mathrm{~A}$ & $47,5 \mathrm{~A}$ & $9,1 \mathrm{~A}$ & $0,91 \mathrm{~B}$ & $10,6 \mathrm{~A}$ \\
\hline $\mathrm{CA}-3,12$ & $4,7 \mathrm{AB}$ & $5,6 \mathrm{~A}$ & $44,7 \mathrm{~A}$ & $9,3 \mathrm{~A}$ & $0,83 \mathrm{~B}$ & $11,5 \mathrm{~A}$ \\
\hline $\mathrm{CA}-6,25$ & $4,8 \mathrm{AB}$ & $5,6 \mathrm{~A}$ & $45,9 \mathrm{~A}$ & $9,3 \mathrm{~A}$ & $0,93 \mathrm{~B}$ & $10,0 \mathrm{~A}$ \\
\hline $\mathrm{CA}-12,5$ & $4,7 \mathrm{AB}$ & $5,6 \mathrm{~A}$ & $45,7 \mathrm{~A}$ & $9,8 \mathrm{~A}$ & $0,91 \mathrm{~B}$ & $11,3 \mathrm{~A}$ \\
\hline $\mathrm{CA}-25$ & $4,4 \mathrm{AB}$ & $5,4 \mathrm{AB}$ & $44,3 \mathrm{~A}$ & $10,3 \mathrm{~A}$ & $0,92 \mathrm{~B}$ & $11,4 \mathrm{~A}$ \\
\hline $\mathrm{CA}-50$ & $4,3 \mathrm{BC}$ & $5,3 \mathrm{AB}$ & $44,6 \mathrm{~A}$ & $9,7 \mathrm{~A}$ & $0,82 \mathrm{~B}$ & $11,8 \mathrm{~A}$ \\
\hline CA - 100 & 4,4 B & $5,2 \mathrm{AB}$ & $45,3 \mathrm{~A}$ & $9,6 \mathrm{~A}$ & $0,82 \mathrm{~B}$ & $12,0 \mathrm{~A}$ \\
\hline $\mathrm{CM}$ & $4,6 \mathrm{AB}$ & $5,4 \mathrm{AB}$ & $43,8 \mathrm{~A}$ & $8,9 \mathrm{~A}$ & $0,95 \mathrm{~B}$ & $9,4 \mathrm{~A}$ \\
\hline testemunha & $4,7 \mathrm{AB}$ & $5,5 \mathrm{~A}$ & $47,1 \mathrm{~A}$ & $9,7 \mathrm{~A}$ & $0,83 \mathrm{~B}$ & $11,9 \mathrm{~A}$ \\
\hline F. blocos & $0,85^{\mathrm{NS}}$ & $0,88^{\mathrm{NS}}$ & $2,39^{\mathrm{NS}}$ & $0,95^{\mathrm{NS}}$ & $9,63 * *$ & $11,34 * *$ \\
\hline F. Trat. & $7,89 * *$ & $2,85 *$ & $1,58^{\mathrm{NS}}$ & $1,58^{\mathrm{NS}}$ & $4,32 * *$ & $2,76 * *$ \\
\hline $\mathrm{CV}(\%)$ & 4,3 & 4,17 & 3,43 & 6,45 & 12,78 & 15,51 \\
\hline DMS & 0,58 & 0,67 & 4,67 & 1,82 & 0,36 & 4,78 \\
\hline
\end{tabular}

${ }^{1}$ Dados transformados para arc sen $\sqrt{\mathrm{x}}$; DAA $=$ dias após a aplicação.

${ }^{\text {NS }}$ não-significativo pelo teste $\mathrm{F} ; *$ significativo a $5 \%$ de probabilidade; ** significativo a $1 \%$ de probabilidade.

Tabela 6 - Porcentagens de frutos abortados no decorrer do período experimental e número acumulado aos 120 DAA, em função dos tratamentos aplicados em frutos de até $2 \mathrm{~cm}$ de diâmetro. Taiúva, 1998

\begin{tabular}{|c|c|c|c|c|c|c|c|c|}
\hline \multirow{2}{*}{ Tratamentos } & \multicolumn{6}{|c|}{ Porcentagem de aborto de frutos } & \multicolumn{2}{|c|}{ Número final } \\
\hline & $15 \mathrm{DAA}$ & $30 \mathrm{DAA}$ & 45 DAA & 60 DAA & $90 \mathrm{DAA}$ & $120 \mathrm{DAA}$ & Orig. & Transf. $^{1}$ \\
\hline C $-3,12$ & $1,9 \mathrm{~A}$ & $1,9 \mathrm{~A}$ & $0,0 \mathrm{~A}$ & 0 & $0,0 \mathrm{~B}$ & $0,0 \mathrm{~A}$ & 1,0 & $2,6 \mathrm{BC}$ \\
\hline$C-6,25$ & $3,8 \mathrm{~A}$ & $3,8 \mathrm{~A}$ & $0,0 \mathrm{~A}$ & 0 & $0,0 \mathrm{~B}$ & $0,0 \mathrm{~A}$ & 2,0 & $4,7 \mathrm{ABC}$ \\
\hline$C-12,5$ & $5,7 \mathrm{~A}$ & $1,9 \mathrm{~A}$ & $0,0 \mathrm{~A}$ & 0 & $0,0 \mathrm{~B}$ & $0,0 \mathrm{~A}$ & 2,3 & $5,0 \mathrm{AB}$ \\
\hline$C-25$ & $7,3 \mathrm{~A}$ & $3,8 \mathrm{~A}$ & $0,0 \mathrm{~A}$ & 0 & $0,0 \mathrm{~B}$ & $0,0 \mathrm{~A}$ & 3,6 & $6,3 \mathrm{AB}$ \\
\hline$C-50$ & $1,9 \mathrm{~A}$ & $6,6 \mathrm{~A}$ & $0,0 \mathrm{~A}$ & 0 & $0,0 \mathrm{~B}$ & $0,0 \mathrm{~A}$ & 2,6 & $5,0 \mathrm{AB}$ \\
\hline C -100 & $0,0 \mathrm{~A}$ & $6,0 \mathrm{~A}$ & $4,6 \mathrm{~A}$ & 0 & $4,6 \mathrm{~A}$ & $1,9 \mathrm{~A}$ & 4,0 & $6,6 \mathrm{~A}$ \\
\hline $\mathrm{CA}-1,06$ & $5,4 \mathrm{~A}$ & $4,6 \mathrm{~A}$ & $0,0 \mathrm{~A}$ & 0 & $0,0 \mathrm{~B}$ & $0,0 \mathrm{~A}$ & 2,0 & $4,6 \mathrm{ABC}$ \\
\hline $\mathrm{CA}-3,12$ & $0,0 \mathrm{~A}$ & $1,9 \mathrm{~A}$ & $0,0 \mathrm{~A}$ & 0 & $0,0 \mathrm{~B}$ & $0,0 \mathrm{~A}$ & 0,3 & $1,1 \mathrm{C}$ \\
\hline $\mathrm{CA}-6,25$ & $1,9 \mathrm{~A}$ & $5,7 \mathrm{~A}$ & $0,0 \mathrm{~A}$ & 0 & $0,0 \mathrm{~B}$ & $0,0 \mathrm{~A}$ & 1,6 & $4,0 \mathrm{ABC}$ \\
\hline $\mathrm{CA}-12,5$ & $1,9 \mathrm{~A}$ & $1,9 \mathrm{~A}$ & $0,0 \mathrm{~A}$ & 0 & $0,0 \mathrm{~B}$ & $0,0 \mathrm{~A}$ & 1,0 & $2,6 \mathrm{BC}$ \\
\hline $\mathrm{CA}-25$ & $0,0 \mathrm{~A}$ & $6,5 \mathrm{~A}$ & $0,0 \mathrm{~A}$ & 0 & $0,0 \mathrm{~B}$ & $0,0 \mathrm{~A}$ & 1,3 & $3,7 \mathrm{ABC}$ \\
\hline $\mathrm{CA}-50$ & $1,9 \mathrm{~A}$ & $8,5 \mathrm{~A}$ & $1,9 \mathrm{~A}$ & 0 & $0,0 \mathrm{~B}$ & $0,0 \mathrm{~A}$ & 3,3 & $6,0 \mathrm{AB}$ \\
\hline CA - 100 & $1,9 \mathrm{~A}$ & $8,1 \mathrm{~A}$ & $1,9 \mathrm{~A}$ & 0 & $1,9 \mathrm{AB}$ & $2,7 \mathrm{~A}$ & 3,9 & $6,5 \mathrm{~A}$ \\
\hline CM & $1,9 \mathrm{~A}$ & $3,8 \mathrm{~A}$ & $0,0 \mathrm{~A}$ & 0 & $0,0 \mathrm{~B}$ & $0,0 \mathrm{~A}$ & 1,3 & $3,7 \mathrm{ABC}$ \\
\hline testemunha & $2,7 \mathrm{~A}$ & $0,0 \mathrm{~A}$ & $0,0 \mathrm{~A}$ & 0 & $0,0 \mathrm{~B}$ & $0,0 \mathrm{~A}$ & 1,4 & $3,8 \mathrm{ABC}$ \\
\hline F. blocos & $0,25^{\mathrm{NS}}$ & $1,82^{\mathrm{NS}}$ & $1,44^{\mathrm{NS}}$ & ----- & $1,25^{\mathrm{NS}}$ & $0,51^{\mathrm{NS}}$ & --- & $5,4 *$ \\
\hline F. trat. & $1,49^{\mathrm{NS}}$ & $1,80^{\mathrm{NS}}$ & $2,01^{\mathrm{NS}}$ & ----- & $2,55 *$ & $0,90^{\mathrm{NS}}$ & ---- & $4,79 * *$ \\
\hline $\mathrm{CV}(\%)$ & 119,45 & 74,94 & 283,7 & ----- & 313,44 & 489,37 & ---- & 28,31 \\
\hline DMS & 9,27 & 9,86 & 4,84 & ----- & 4,13 & 4,56 & ---- & 4,41 \\
\hline
\end{tabular}

${ }^{1}$ Dados transformados para arc sen $\sqrt{x}_{\mathrm{x}}$, DAA $=$ dias após a aplicação.

${ }^{\text {NS }}$ não-significativo pelo teste $\mathrm{F} ; *$ significativo a $5 \%$ de probabilidade; $* *$ significativo a $1 \%$ de probabilidade. 
Tabela 7 - Resultados da análise tecnológica de frutos de laranjeira 'Hamlin' em resposta aos tratamentos, quando aplicados no estádio de frutos de até $2 \mathrm{~cm}$ de diâmetro. Taiúva, 1998

\begin{tabular}{|c|c|c|c|c|c|c|}
\hline \multirow{2}{*}{ Tratamentos } & \multicolumn{2}{|c|}{ Diâmetro $(\mathrm{cm})$} & \multirow{2}{*}{$\%$ Suco $^{1}$} & \multirow{2}{*}{${ }^{\circ}$ Brix } & \multirow{2}{*}{ Acidez titulável } & \multirow{2}{*}{ Ratio } \\
\hline & $150 \mathrm{DAA}$ & 240 DAA & & & & \\
\hline $\mathrm{C}-3,12$ & $4,7 \mathrm{AB}$ & $5,5 \mathrm{ABCD}$ & $46,1 \mathrm{~A}$ & $9,9 \mathrm{~A}$ & $0,85 \mathrm{~A}$ & $11,7 \mathrm{~A}$ \\
\hline$C-6,25$ & 4,6 $\mathrm{ABC}$ & 5,5 ABCD & $46,2 \mathrm{~A}$ & $9,6 \mathrm{~A}$ & $0,86 \mathrm{~A}$ & $11,2 \mathrm{~A}$ \\
\hline$C-12,5$ & $4,5 \mathrm{ABC}$ & 5,5 ABDC & $45,3 \mathrm{~A}$ & $9,6 \mathrm{~A}$ & $0,82 \mathrm{~A}$ & $11,7 \mathrm{~A}$ \\
\hline$C-25$ & $4,5 \mathrm{ABC}$ & $5,7 \mathrm{ABC}$ & $46,2 \mathrm{~A}$ & $9,7 \mathrm{~A}$ & $0,89 \mathrm{~A}$ & $10,9 \mathrm{~A}$ \\
\hline$C-50$ & $4,3 \mathrm{BC}$ & $5,3 \mathrm{BCD}$ & $45,8 \mathrm{~A}$ & $9,3 \mathrm{~A}$ & $0,85 \mathrm{~A}$ & $10,4 \mathrm{~A}$ \\
\hline C -100 & $3,4 \mathrm{D}$ & $4,3 \mathrm{E}$ & $42,4 \mathrm{~B}$ & $10,9 \mathrm{~A}$ & $1,00 \mathrm{~A}$ & $11,0 \mathrm{~A}$ \\
\hline $\mathrm{CA}-1,06$ & $4,6 \mathrm{ABC}$ & $5,6 \mathrm{ABC}$ & $44,5 \mathrm{AB}$ & $10,2 \mathrm{~A}$ & $0,88 \mathrm{~A}$ & $11,5 \mathrm{~A}$ \\
\hline $\mathrm{CA}-3,12$ & $4,7 \mathrm{AB}$ & 5,5 $\mathrm{ABCD}$ & $45,5 \mathrm{AB}$ & $10,3 \mathrm{~A}$ & $0,87 \mathrm{~A}$ & $11,9 \mathrm{~A}$ \\
\hline $\mathrm{CA}-6,25$ & $4,9 \mathrm{~A}$ & $5,7 \mathrm{AB}$ & $45,6 \mathrm{~A}$ & $10,5 \mathrm{~A}$ & $0,89 \mathrm{~A}$ & $11,9 \mathrm{~A}$ \\
\hline $\mathrm{CA}-12,5$ & $4,9 \mathrm{~A}$ & $5,8 \mathrm{~A}$ & $45,3 \mathrm{AB}$ & $10,0 \mathrm{~A}$ & $0,86 \mathrm{~A}$ & $11,7 \mathrm{~A}$ \\
\hline $\mathrm{CA}-25$ & 4,6 $\mathrm{ABC}$ & 5,5 ABCD & $45,2 \mathrm{AB}$ & $10,6 \mathrm{~A}$ & $1,03 \mathrm{~A}$ & $10,7 \mathrm{~A}$ \\
\hline $\mathrm{CA}-50$ & $4,4 \mathrm{ABC}$ & $5,3 \mathrm{BCD}$ & $47,5 \mathrm{~A}$ & $10,1 \mathrm{~A}$ & $0,89 \mathrm{~A}$ & $11,5 \mathrm{~A}$ \\
\hline CA - 100 & $4,1 \mathrm{C}$ & $5,1 \mathrm{D}$ & $46,3 \mathrm{~A}$ & $9,8 \mathrm{~A}$ & $0,86 \mathrm{~A}$ & $10,3 \mathrm{~A}$ \\
\hline $\mathrm{CM}$ & $4,3 \mathrm{BC}$ & $5,2 \mathrm{CD}$ & $46,2 \mathrm{~A}$ & $9,7 \mathrm{~A}$ & $0,85 \mathrm{~A}$ & $11,5 \mathrm{~A}$ \\
\hline testemunha & $4,9 \mathrm{~A}$ & $5,6 \mathrm{ABC}$ & $45,7 \mathrm{~A}$ & $10,5 \mathrm{~A}$ & $0,98 \mathrm{~A}$ & $10,7 \mathrm{~A}$ \\
\hline F. blocos & $5,67 * *$ & $1,07^{\mathrm{NS}}$ & $2,79^{\mathrm{NS}}$ & $1,42^{\mathrm{NS}}$ & $0,91^{\mathrm{NS}}$ & $0,37^{\mathrm{NS}}$ \\
\hline F.Trat. & $12,59 * *$ & $16,23 * *$ & $3,43 * *$ & $1,61^{\mathrm{NS}}$ & $2,21 *$ & $0,66^{\mathrm{NS}}$ \\
\hline $\mathrm{CV}(\%)$ & 4,06 & 2,81 & 2,26 & 6,17 & 7,91 & 10,1 \\
\hline DMS & 0,55 & 0,46 & 3,12 & 1,88 & 2,14 & 3,44 \\
\hline
\end{tabular}

Com relação à mistura de clomazone com ametryn, verificou-se o mesmo comportamento de resposta à dose utilizada, porém com sintomas se manifestando a partir da dose de $12,5 \%(\mathrm{CA}-12,5)$ e mais acentuadamente nas folhas do que nos frutos. Embora as menores doses dos dois produtos também tenham causado sintomas de clorose nas folhas e/ou nos frutos, esses foram menos intensos e perduraram até no máximo 45 DAA, enquanto sintomas decorrentes da aplicação das maiores doses persistiram até 60-90 DAA, culminando com a queda dos frutos no tratamento empregando $100 \%$ de clomazone isolado.

Com a formulação microencapsulada do clomazone (CM), aplicado na dose comercial, observou-se redução nos sintomas de fitotoxicidade, com a clorose se manifestando principalmente nos frutos, porém em intensidade muito menor do que a mesma dose na formulação de concentrado emulsionável, sem, entretanto, resultar no aborto de frutos e com suas folhas se assemelhando às da testemunha. Verificou-se, ainda, que a aplicação da maior dose de clomazone isolado ou em mistura com ametryn em frutos já formados resultou em manchas cloróticas e/ou necróticas, mais acentuadamente nos tratados com a mistura, que se manifestaram com o desenvolvimento dos frutos, principalmente na face exposta à luz solar, enquanto nos frutos da testemunha não foram observados quaisquer sintomas semelhantes.

Quando a simulação da deriva foi feita com a maior dose de clomazone (C-100) e com as duas maiores doses dele em mistura com ametryn (CA-50 e CA-100) e a planta encontravase em pleno crescimento vegetativo, ou seja, com emissão de novos ramos, estes, além de apresentarem as folhas totalmente cloróticas, decorridos de 60 a 90 DAA, caíram e entraram em processo de senescência, secando por completo após 100 DAA.

Por ocasião da colheita, verificou-se que os frutos tratados com a maior dose de clomazone, além de apresentarem as manchas necróticas, também apresentavam menor diâmetro do que os frutos da testemunha, enquanto 
naqueles que foram tratados com clomazone em mistura com ametryn a formação dessas manchas necróticas foi mais perceptível do que a redução dos diâmetros dos frutos em si.

A formulação de clomazone microencapsulado, embora não tenha causado a formação de manchas necróticas, provocou redução no tamanho dos frutos, a qual foi bem menos acentuada do que a causada pelo mesmo produto na formulação de concentrado emulsionável.

Os sintomas de intoxicação das plantas de laranja pelo clomazone, caracterizados pela formação de manchas cloróticas nas folhas mais novas, que com o desenvolvimento das folhas foram coalescendo até que estas ficassem totalmente despigmentadas, com conseqüente morte dessas, estão de acordo com o seu mecanismo de ação para o controle de plantas daninhas. Segundo a FMC (s.d.) e Rodrigues \& Almeida (1998), o clomazone inibe a síntese de compostos isoprenóides, que são os precursores de pigmentos fotossintéticos, causando redução no nível de caroteno e fitol e, conseqüentemente, clorofila. Uma vez que o caroteno protege a clorofila da destruição pela luz solar, o mecanismo de ação do produto torna-se bidirecional, inibindo a produção de clorofila e de pigmentos protetores desta. Com isso, as folhas ficam descoloridas, por falta de clorofila, morrendo em pouco tempo.

A absorção do produto ocorre preferencialmente pelo meristema apical da planta, e uma vez que sua translocação ocorre pelo xilema, seguindo o fluxo transpiracional, os sintomas de sua intoxicação em plantas deverão se manifestar nos órgãos mais novos (Rodrigues \& Almeida, 1998).

Contudo, os sintomas de intoxicação pelo clomazone que se manifestaram nos frutos, com a formação de manchas cloróticas e/ou necróticas, tenha sido ele aplicado isolado ou em mistura com ametryn, assim como o produto causando a mortalidade de ramos, não haviam ainda sido relatados. Também, não foi encontrado na literatura qualquer trabalho que relatasse os efeitos da deriva do clomazone, simulada ou não, sobre a porcentagem de aborto de flores/frutos de laranjeira, assim como sobre as características qualitativas por meio de análise tecnológica dos frutos.
Bode (1984) afirma que a deriva de herbicidas é complexa, envolvendo a interação de vários fatores, como os bicos utilizados, características do líquido pulverizado, condições climáticas e deposição de gotas, entre outras. Isso faz com que seja difícil determinar com precisão o grau de deriva que ocorre, com conseqüente queda na produtividade (Lyon \& Wilson, 1986). Além disso, Russo (1990) afirma que a deriva pode ser um evento único ou múltiplo, quando as plantas são atingidas várias vezes pelos produtos, em parte devido às correntes circulatórias de ar.

No caso de aplicação com equipamento terrestre, Grover et al. (1972) e Hatterman-Valenti et al. (1995) afirmam que cerca de 1 a $8 \%$ do volume de líquido aplicado se deposita fora da faixa de aplicação. Por outro lado, de acordo com Schweizer (1978), à medida que se aumenta a distância em relação à faixa de aplicação, decrescem os danos ocasionados pela deriva, bem como sua concentração.

Segundo Bode (1984), resultados de 80 ensaios demonstram que em pulverizações de herbicidas com equipamentos terrestres, em condições de ventos com velocidade de 4,4 $\mathrm{ms}^{-1}$, a deposição de produtos devido à deriva situouse em torno de 9,2\%; com razoável cuidado na aplicação, a deriva pode ser reduzida a 5\% da dose aplicada, e, utilizando alta tecnologia, foi possivel obter deriva inferior a $2 \%$. O mesmo autor afirma que, de forma generalizada, a deriva situa-se em torno de 3 a $5 \%$ do produto aplicado, com aproximadamente 50\% dela se depositando nos primeiros 15 metros, decaindo rapidamente à medida que aumenta a distância em relação à faixa de aplicação.

Para Dexter (1979), citado por Schroeder et al. (1983), a detecção da ocorrência de deriva na aplicação de herbicidas tem grande importância, pois, enquanto as perdas econômicas ocasionadas por este fato não forem facilmente identificadas, alguns agricultores que cultivam plantas suscetiveis em áreas adjacentes à de aplicação podem ter substanciais reduções na produção, sem no entanto identificar a verdadeira causa. Segundo Auch \& Arnold (1978), existem diferenças de tolerância entre os diferentes estádios de desenvolvimento da planta, entre diferentes espécies e/ou variedades, aos herbicidas. 
De acordo com Hemphill Júnior \& Montgomery (1981), os efeitos de doses letais de herbicidas sobre as folhas das plantas são conhecidos. Entretanto, os efeitos da exposição a doses não-letais, o que pode ocorrer em função da deriva, são poucos conhecidos. Em virtude do relatado, pode-se inferir que:

- A simulação de deriva de clomazone em ramos de laranjeira, cv. Hamlin, florescidos e com frutos já formados de até $2 \mathrm{~cm}$ de diâmetro, resultou em aborto de frutos apenas quando foi utilizada a dose comercial, não sendo constatado nenhum efeito de aborto quando em mistura com ametryn ou na formulação microencapsulada. Além disso, observou-se que a concentração a partir de $25 \%$ da dose comercial de clomazone isolado e acima de 50\% em mistura com ametryn, bem como na formulação microencapsulada, resultou em frutos com menor diâmetro.

- A simulação de deriva de clomazone, isolado ou em mistura, e na formulação microencapsulada, até as suas doses comerciais, não acarretou qualquer alteração quanto à análise tecnológica dos frutos.

- As doses acima de 50\% de clomazone isolado e em mistura com ametryn levaram a formação de manchas cloróticas e/ou necróticas na casca do fruto.

\section{REFERÊ NCIAS BIBLIOGRÁFICAS}

AUCH, D.E., ARNOLD, W.E. Dicamba use and injury on soybeans (Glycine max) in South Dakota. Weed Sci., v.26, p.471-475, 1978.

BAYLEY, J.A., KAPUSTA, G. Soybean (Glycine max) tolerance to simulated drift of nicosulfuron and primisulfuron. Weed Technol., v.7, n.3, p.740745. 1993.

BODE, L.E. Downwind drift deposits by ground applications. In: PESTICIDE DRIFT MANAGEMENT SYPOSIUM, 1984, Brookings, SD. Proceedings... Brookings, SD: South Dakota University. 1984. p.49-52.

DURIGAN, J.C. Comportamento de herbicidas no ambiente In: SEMINÁRIO TÉCNICO SOBRE PLANTAS DANINHAS E O USO DE HERBICIDAS EM REFLORESTAMENTO, 1989, Rio de Janeiro. Anais... Rio de Janeiro: SBS/ABRACAVE/SIF, 1989. Paginação irregular.
FIGUEIREDO, J.O. Variedades de copa de valor comercial. In: RODRIGUEZ, O., VIÉGAS, F., POMPEU JR., J., AMARO, A.A. Citricultura Brasileira. 2.ed. Campinas: Fundação Cargill, 1991. v.1, p.234.

FMC. Manual de produtos. São Paulo: [19 --] p.67$75,83-87$.

GELMINI, G.A. Herbicidas: indicações básicas. Campinas: Fundação Cargill, 1988. 334p.

GROVER, R., MAYBANK, J., YOSHIDA, K. Droplet and vapor drift from butyl ester and dimethylamine salt of 2,4-D. Weed Sci., v.20, n.4, p.320-4, 1972 .

HATTERMAN-VALENTI, H., OWEN, M.D.K., CHRISTIANS, N.E. Comparison of spray drift during postemergence herbicide applications to turfgrass. Weed Technol., v.9, n.2, p.321-325, 1995.

HEMPHILL JÚNIOR., D.D., MONTGOMERY, M. L. Response of vegetable crops to sublethal application of 2,4D. Weed Sci., v.29, p.632-635, 1981.

LYON, D.J., WILSON, R.G. Sensitivity of fieldbeans (Phaseolus vulgaris L.) to reduced rates of 2,4D and dicamba. Weed Sci., v.34, p.953-956, 1986.

MATUO, T. Técnicas de aplicação de defensivos agrícolas. Jaboticabal: FUNEP, 1990. 136p.

RUSSO, V.M. Reaction of tomato cultivars to a sublethal dose of glyphosate. Hortscience, v.25, n.12, p.1662, 1990.

RODRIGUES, B.N., ALMEIDA, F.S. Guia de herbicidas. 4.ed. Londrina: Edição dos autores, 1998. 648p.

SCHROEDER, G.L., COLE, D.F., DEXTER, A.G. Sugarbeet (Beta vulgaris L.) response to simulated herbicide spray drift. Weed Sci., v.31, p.831-36, 1983.

SCHWEIZER, E. E. Response of sugarbeets (Beta vulgaris) to sublethal rates of 2,4-D. Weed Science, v.26, p. 629-631, 1978.

TRESSLER, D.K., JOSLYN, M.A. Fruits and vegetable juice: - processing technology. Westport: The AVI Publications, 1961. 1028p.

YATES, W.E., AKESSON, N.B., BAYER, D.E. drift of glyphosate sprays applied with aerial and ground equipment. Weed Sci., v.26, n.6, p.597604, 1978. 